\title{
Evidence-based Reconstruction of Kampo Medicine: Part II-The Concept of Sho
}

\author{
Katsutoshi Terasawa
}

Toyama Medical and Pharmaceutical University, Japan

\section{Introduction}

In this lecture, I would like to focus on and discuss what I think is the central concept of Kampo: the concept of Sho. It can be roughly translated into English as, for example, symptoms, signs or evidence, and I shall try to explain (though not define) it in more detail in the second section of this article. There are several reasons why I try to represent Kampo practice as based centrally on Sho, besides the fact that I believe it is really part of the essence of Kampo.

First, many Western scientists and physicians are put off from the start if they are told that it is impossible for them to understand traditional Chinese medicine (TCM) or Kampo without grasping the full complexities of its basic concepts such as Ying-Yang, five elements, five organs, Qi, etc. If Western scientists and physicians are told that the TCM paradigm is so different from modern Western medicine that they should first switch the paradigm itself, they will be hopeless when they try to come to terms with any specific Kampo or TCM remedies. In some sense this is true and it is very risky for Western physicians to pick up any Kampo or TCM formulae outside the context of Kampo's cognitive paradigm. However, since saying that the paradigm should come first may only result in shutting the door against those who genuinely wish to enter the rich field of TCM or Kampo, I would think that it is more productive to first focus on what I think is

For reprints and all correspondence: Professor Katsutoshi Terasawa, Department of Japanese-Oriental (Kampo) Medicine. Faculty of Medicine, Toyama Medical and Pharmaceutical University.

E-mail: terasawa@ms.toyama-mpu.ac.jp

Professor Katsutoshi Terasawa was born in Tokyo in 1944. He graduated in 1970 from the School of Medicine at Chiba University. He became Director of the Department of Japanese Oriental Medicine at Toyama Medical and Pharmaceutical University in 1979. He was Director of the School of Medicine (1999-2000) and then President of the Hospital and Vice-President (2002-2004) of Toyama Medical and Pharmaceutical University. He is now President of the Japan Society for Oriental Medicine and Director of the WHO Collaborating Center for Traditional Medicine. the central medical concept of Kampo without going any deeper into its paradigm, philosophical foundations or the system itself.

Secondly, I have to emphasize that TCM and Kampo are not monolithic ideological systems. They are broad systems of medical practice and thinking evolved over thousands of years. Therefore, the systems themselves have a rather wide variation. There have been many schools in TCM and Kampo and disputes among them are the rule, rather than the exception. Basic concepts like Yin-You (Ying-Yang in Chinese) or Ki (Qi in Chinese) have been held invariantly among different schools, but slightly different interpretations are common, even for such central concepts as Ying-Yang, and no less common are different emphases on more peripheral concepts. In the first article of this lecture series (1), I pointed out that Kampo could be seen as a simplified or practical version of TCM. Stated differently, Kampo is a sort of school or an important variant of TCM. In that article I stated that modern Kampo in Japan can be traced back to the 18th century Renaissance (or restoration) movement of the Koho (old formula) school of, for example, Yamawaki and Yoshimasu. Western readers may be astonished to know that Yoshimasu went so far as to suggest the irrelevance of the very concept of Ying-Yang, emphasizing, in effect, that anything should go as long as it is effective, regardless of philosophy. Though I should hastily add that such attitudes did not become orthodox in Japan, it cannot be denied that Kampo is less rigorous about its philosophical or ideological basis than the orthodox TCM. Thus, if I were asked by outsiders what the central concept held as consensus among Kampo practitioners in Japan is, I would say that it is Sho.

Thirdly, it is very interesting to highlight the concept of Sho in this journal, Evidence-based CAM, because the Chinese character representing Sho can be translated in English to mean evidence. Also of interest is that the same word may have been differently represented in ancient Chinese character, the meaning of which in turn could be translated in English to

The online version of this article has been published under an open access model. Users are entitled to use, reproduce, disseminate, or display the open access version of this article provided that: the original authorship is properly and fully attributed; the Journal and Oxford University Press are attributed as the original place of publication with the correct citation details given; if an article is subsequently reproduced or disseminated not in its entirety but only in part or as a derivative work this must be clearly indicated. 
mean symptom. Thus, the term Sho bears very interesting medical implications, unlike Ying-Yang or Qi, which would be of interest to any Western scientist or physician who wished to acquire medical knowledge about Kampo or TCM.

Finally, there is a very practical reason peculiar to the Japanese situation surrounding Kampo. This is related to the government's policy on prescriptive drugs. In Japan, it is not possible for a physician to use TCM's three elements/eight categories interpretations in order for his/her Kampo formulae to be covered by the National Health Insurance System (NHIS). Thus, a Kampo formula, if it is to be covered by the NHIS, must be prescribed for disease entities expressed in terms of modern Western medicine, such as gastritis or atopic eczema. For rigorous practitioners of TCM this would be a great insult, as their diagnostic interpretations are not recognized as official or scientific categories of diseases. Thus, nowadays it has become more common for Japanese medical doctors to prescribe Kampo formula just by saying that the patient now has a condition which requires such formula. Though it has long been legitimate in Kampo to prescribe a formula as the patient is simply in its Sho, this tendency has become more prominent these days. For example, a patient can just be said to be Rikkunshi-To-Sho, regardless of his/her diagnosis in terms of modern western medicine. As I shall explain later, this situation arises in the Kampo system itself, where any symptoms (Sho) can be taken as evidence (Sho, again) for some formulae, and very often symptoms are called Sho-for-some Kampo formulae. As drugs covered by NHIS can only be prescribed by medical doctors trained in Western medicine in Japan, there is a tendency for them to use Kampo formula without paying much attention to the TCM interpretations of the symptoms of the patient. Therefore, I think the mainstream of Kampo is Shooriented, meaning that it does not rely so much on the rigorous interpretation of the disease state in terms of the basic TCM concepts as on the direct practical effects of a formula itself.

\section{Overview of the Basic Attitudes/ Concepts of Kampo}

After explaining that I am not much inclined at this moment to go deeper into the paradigmatic or philosophical aspects of Kampo or TCM, I think I would like to introduce the readers to the basic attitudes and concepts of Kampo. I have no intention to be comprehensive or rigorous here; all I wish is to look at the Kampo medical system from the viewpoint of its interested, and not hostile, outsiders probably living in the West.

\section{Qualitative and Intuitive Approach to Individual Patients, Shi-Shin (Four Diagnostic Approaches), or How Sho is Encountered}

One way to come to terms with the Kampo approach is to imagine that, supposing you are a physician, you are faced with a patient in agony, but are deprived of any advanced medical technology, from stethoscope to MRI. You are capable only of physical examinations, but without a stethoscope, thermometer or sphygmomanometer. Interviewing and old fashioned sensual examinations are all that you can perform. As ancient Kampo physicians abhor physical invasions into a patient's body, examinations such as biopsy or endoscopy are unimaginable. So, the only approaches left to you are: (i) visual observation, (ii) listening to the sounds made by the patient's body, (iii) smelling and touching the patient and (iv) listening to what they say. In TCM and Kampo these are called Shi-Shin, which translates to four diagnostic approaches. Of course, some of them have counterparts like inspection and palpation even in today's Western medicine. However, it is hard for a modern physician to imagine such a situation where he/she is allowed only these four approaches. The information he/she will be getting is basically qualitative. It is also not analytical, in the sense that the precise anatomical or biochemical basis of any physiological event would have been unknown to ancient Kampo doctors.

It is difficult to characterize what is achieved by such an approach, but we may say that those pre-technological physicians would only be able to concentrate on purely phenomenal events. A patient is not regarded as a vector of multidimensional quantitative parameters but as an individual with mind-body unity. His/her disease states are seen in a constantly changing flow, not as a bundle of digital information. Such an intuitive or phenomenological diagnostic approach would clearly be insufficient to penetrate deep into the events taking place at cellular or molecular levels, at least analytically. However, it would have great power in grasping the general state of the patient's conditions as a whole. The situation can be compared to ancient and modern weather forecasting. The modern weather forecast, relying much on the analysis of numerical data from satellites, is of course in possession of great power, but it would be a great mistake to assume that ancient people were incompetent in forecasting the weather. Accumulating pure experience over thousands of years, they would have acquired intuitive power to predict the changes of weather just by careful observation of the movement of the clouds, changes in the wind and sensitivity to subtle changes of temperature and moisture. Likewise, Kampo practitioners paid great attention to the subtle changes in the feeling of the pulse or the abdomen of one individual patient. What they obtained were phenomenal, intuitive and qualitative signs, or presentations of one patient's illness, just as ancient weather tellers could gauge the signs of rain from clouds and winds. The term Sho has some connotation as signs; thus, Sho is the first of what is obtained as a result of this purely observational approach.

\section{Major Categories of Disease Conditions and Substances to Illustrate Sho as Symptom in Kampo}

TCM and Kampo developed a characteristic system to categorize or explain Sho. As I said above, this is sometimes a great stumbling block, and sometimes a source of mysterious charm, for those Westerners who wish to understand TCM or Kampo. It should be remembered that the factual basis of the 
system of Kampo or TCM is as described above: it is phenomenological, intuitive and qualitative. The strangeness (or mystery) of the system seen from the modern Western viewpoint would become partially understandable if one thought of its experiential basis.

As Kampo judgment is basically intuitive, there is great danger in trying to verbally define its system concepts, and no less misleading would be to try to translate them into different languages, into English in the present case. So, although in this section I would like to briefly explain some of the basic Kampo system concepts on which Sho is founded, these should not be regarded as strict definitions in the traditional Western philosophical sense. I would rather suggest that they are only guidelines for understanding, and thus anyone who would really like to understand the meaning of these concepts should learn them from Kampo masters through actual clinical experience.

I think one good way for Westerners to look at the Kampo system is that there are essentially four (three) dichotomic categories and three substance concepts in which Sho is understood as symptom. The three dichotomies are: Yin-You (Ying-Yang), Kyo-Jitsu and Netsu-Kan. They can roughly be represented as positive-negative, hollow-full and hot-cold, respectively, in English. There are several more topological categories like Hyou-Ri (surface-underside, or maybe explicit-covert), but in this lecture I would like to omit them.

\section{Disease Condition (or States) Categories: Yin-Yo (Ying-Yang), Jitsu-Kyo and Kan-Netsu}

\section{A. Yin Sho-Yo Sho}

This would be the most famous concept not only for Kampo or TCM, but for Chinese thought or philosophy in general. This concept is probably derived from bright-dark dichotomy in ancient China, but can also denote such varieties of pairconcepts as positive-negative, convex-concave, active-passive, male-female, plus-minus, etc. In medical terms, if the repairing responses shown by the patient against his/her disease condition are feverish, active or excitatory, the patient is said to be in Yo-Sho. Conversely, if the responses shown by the patient are chilly, inactive or inhibitory, the patient is said to be in Yin-Sho.

\section{B. Jitsu Sho-Kyo Sho}

This dichotomy is rather abstract and could be represented as real-unreal, but is rooted probably in the intuitive concept of full/hollow or strong/weak. Thus, if the repairing responses shown by the patient against his/her disease condition are strong or fully active, the patient is said to be in Jitsu-Sho, while if they are weak or hollow, they are said to be in Kyo-Sho.

\section{Netsu Sho-Kan Sho}

This is probably the most concrete dichotomy derived from the experience of febrile illness of ancient ages. Thus, if the repairing responses shown by the patient against his/her disease condition are febrile, the patient is said to be in Netsu-Sho, while if they are chilly the patient is said to be in Kan-Sho.

\section{The Three Substance Categories: Ki, Ketsu and Sui}

In Kampo or TCM, Sho is mainly understood as disturbances in the three major substances of life. They are Ki (Qi), Ketsu and Sui. Of the three, many Westerners would be acquainted with Ki (Qi), through, for example, Qigong. Ki can be understood as energy fundamental to living things, especially for human beings who possess mind or spirit. In Kampo or TCM, life functions of human beings are thought to be controlled by $\mathrm{Ki}$. I would like to stress that in (East) Asian outlook where mind/body dichotomy is rather alien, $\mathrm{Ki}$ is regarded as the energy of a unified mind-body. Therefore, though there seems to be no other option than representing it as spirit in English, Western readers are warned against interpreting this to be pure spirit. For example, a person in good spirits but in very bad physical condition is not usually seen to be in excellent $\mathrm{Ki}$ condition in China and Japan.

In contrast to the Ki concept, Ketsu and Sui are more material and probably much closer to the usual concepts of blood and body fluids, respectively, in Western terms. Without any precise knowledge of cellular/molecular physiology, ancient TCM physicians would have thought blood and body fluids to be two major substances. In modern Kampo, these concepts are still used, in broader overlap with blood and body fluids in modern terms.

\section{Some Examples of Sho Expressed in Disease Conditions (States) and Substances}

There are several major Shos expressed in terms of the above categories. The following are not an exhaustive list, but just some examples to convey to Western readers how Sho is expressed in terms of these categories.

\section{A. Ki-Kyo and Ketsu-Kyo}

Kampo physicians sometimes see a patient's symptoms as $\mathrm{Ki}-\mathrm{Kyo}$, or maybe hollow-spirited, meaning that the patient's $\mathrm{Ki}$ is not sufficient (strong) enough to sustain his/her living functions. For example, fatigue, drowsiness or appetite loss in Western terms, are often seen as Ki-Kyo in Kampo terms. Likewise, deficiency in Ketsu (blood), said to be Ketsu$\mathrm{Kyo}$, is often seen in pallor, dizziness or alopecia in Western terms.

\section{B. Ki-utsu and $\mathrm{Ki}$-gyaku}

Utsu literally means stagnation, while gyaku means counteraction. Thus, a patient's symptoms are sometimes seen as caused by stagnation of $\mathrm{Ki}$, for example, in depression, heavyheadedness or nausea in Western terms. Likewise, a patient is seen as showing Sho of Ki-gyaku, or with counter-circulated $\mathrm{Ki}$, for example, in palpitations, irritation or paroxysmal headache in Western terms. 


\section{O-Ketsu and Sui-Tai}

O-Ketsu refers to the condition of stagnation of blood, which is sometimes seen in such conditions as lower limb varix, teleangiectasis and pigmentation of the skin. Sui-tai in turn refers to the condition of stagnation of body fluids, which is sometimes seen in such conditions as dizziness, edema and swelling of the tongue.

\section{On Anatomical and Disease Phase Concepts}

Kampo and TCM have peculiar anatomical concepts (five solid and six hollow organs) and six disease phase concepts, like major-Yang, minor-Yang and minor-Yin phases. However, I shall leave explanations for these for later articles in this series as they become necessary, in order not to confuse readers.

\section{A Simplified Representation of the Kampo Approach}

Based on the previous discussions, I think it would be profitable for Western readers to understand the Kampo approach as simplified below.

A Kampo doctor basically views a diseased state of a patient as deviation from the optimal balance. As seen in the previous section, all the four categories can be viewed as deviation to two extremes: for example, Ying may mean too dark or too negative, while Yang may mean too bright or too positive, and Kyo may mean too hollow, while Jitsu may mean too full. It would be basic for the Kampo and TCM perspective to understand the healthy state of human beings as being a wellbalanced or undeviated condition of mind/body.

This well-balanced state is disturbed by the imbalance of the basic three substances of human mind/body. So, for example, a patient may be in a state of too hollow-spirited (Ki-Kyo), or too-congested-blood (O-Ketsu).

The disease conditions thus seen as deviation or imbalance are grasped as such by the four physical approaches (Shi-Shin) as explained above. Without advanced tools or precise anatomical knowledge, Kampo physicians especially emphasized the observations of the abdominal and pulse conditions. There are lots of subtle inspective and palpative notions that can discern various Shos in Kampo, but it is futile to try and explain them in written words; they will only be learned from real experience with Kampo masters.

This is a rough sketch of how Sho is raised, and how each Sho is directly connected with the specific Kampo formula (Hou), which would be prescribed for it. This connection, ShoHo (symptom-formula) correspondence, is characteristic of Kampo medicine.

\section{Sho as the Crucial Concept in Kampo}

\section{How to Understand the Sho Concept}

Up to this point, I hope, Western readers have gained some impression of what Sho is like. It should be first understood negatively as something different from modern disease entities (breast cancer, tuberculosis or rheumatoid arthritis). But it is also different from symptoms, like fever or hematoemesis in modern Western medicine, which is a phenomenal consequence of the disease entity, which is its substantial basis.

Without having the concept of disease entity in the modern sense, ancient TCM/Kampo physicians developed a purely phenomenological concept of Sho. Sho is thus a dual concept expressing both diagnosis and indication for Kampo prescriptions. You can say that Sho is a mixed-up or ambiguous concept: phenomenological subtlety and non-analytical vagueness are two sides of the same coin. Sho is also an intuitive concept of the condition of one patient as a whole, assessed by intuitively and qualitatively classifying him/her as one person in view of three substances and eight categories (four dichotomic categories).

In effect, in classifying a patient in terms of Sho, Kampo physicians see him/her in an imbalanced state toward one or another extreme direction. Kampo herbs are prescribed to correct this imbalance, or help the individual patient turn back to the equilibrium state where Ki, Ketsu and Sui are well balanced in his/her mind/body totality. As Sho is phenomenological, it is changeable. A patient's Sho can be changed in a day or hours. This fluidity or non-rigidity of Sho makes Kampo diagnosis unlike technology, but close to art. Also, because it is phenomenological, Sho cannot be separated from the individual patient. In Western medicine a patient can be a case of lung cancer, but there is no patient as a case of, say, Kyo-sho. The patient presents him/herself as Kyo-sho.

Incidentally, in authentic TCM (mainly based on the neoConfucian ideology of Ki-and-Ri, or maybe Spirit-andReason), much emphasis seems to be placed on verbal arguments about Sho, based not only on four physical approaches, three elements and eight categories, but also on the metaphysical concepts of five elements and anatomical concepts of five solid and six hollow organs. Thus, it may be misleading to represent Sho as purely a phenomenological/ non-analytical concept but, as I sometimes point out, there is a tendency in Japanese Kampo medicine to understand Sho intuitively, without much resort to verbal arguments. This conceptual situation has one important practical consequence. In orthodox TCM, physicians are usually required to argue about Sho-as-symptoms in detail in order to reach Sho-as-formula (or prescription). On the other hand, in current Japanese Kampo, unlike the orthodox TCM of China, it is now quite legitimate to express Sho in terms only of formula. For example, Japanese Kampo physicians often say: the patient is in Rikkunshi-To-Sho. This means that the patient is in a condition in which Rikkunshi-To will be effective.

As you can see, in this paradigm, diagnosis is completely merged with formula. In a Western situation, this would be similar to stating that the patient is in the condition of, say, aspirinSho. Although it may sound very strange at first, you will find that such an approach is sometimes of great advantage. As even for one purely synthetic chemical drug like aspirin, its effects range widely in terms of disease categories diagnosed based on the modern pathophysiology. Aspirin is effective for a wide 
spectrum of disease conditions from common cold and thrombosis, to (prevention of) some gastrointestinal cancers. So we can imagine a hypothetical situation where the biochemical basis for a fever, thrombosis or even some cancer is totally unknown, but an experienced physician could intuitively judge that a patient is aspirin-Sho. While the pathophysiology of such diseases or even the disease entities themselves were not established, the physician would have been regarded as a magician. But biochemical and pharmacological research would later find evidence that the physician's intuition had a firm scientific basis. I am sure that some of the Sho and herbal formulae in Kampo await the discovery of their surprising molecular basis Although the Sho approach of mixed diagnosis-formula was the only pragmatic way to deal with illness when any disease seemed complex and obscure, it can still teach us something even today in dealing with those disease conditions which look complex or are of obscure origin, such as atopic dermatitis or chronic fatigue syndrome.

\section{The Many-to-many Correspondence Between Kampo Sho and Western Disease Entities}

From the discussion above, it should be understood that basically there can be no one-to-one relationship between one Sho and any one symptom, disease or formula of modern Western medicine. The classification system, or the paradigm in the most elementary sense, is completely different.

But, viewed differently, the situation can be seen as many-tomany relationships between Sho and disease. Thus, for any one disease entity, there could be plural Sho. For example, one simple disease state of common cold in Western diagnosis can be in two different Sho states, such as Kakkon-To-Sho and MaouBushi-Saishin-To-Sho, dependent, mainly, upon the Netsu-Sho or Kan-Sho and the state of pulsation. In contrast, many different disease entities in Western terms can show the same, or very similar, Sho in some instances. For example, Dai-Sai-Ko-To has been used in such variety of disease conditions as gastritis, sinusitis, hypertension, cholelithiasis and eczema.

Of course such a many-to-many relationship also holds for symptoms-disease and formula-disease correspondence in the Western paradigm. But, in the Western paradigm, any correspondence between symptoms and disease presupposes an anatomical and pathophysiological basis, and any correspondence between formulae and disease presupposes a biochemical and pharmacological action mechanism, both in the modern sense. Therefore, the correspondence can be traced through such biological strings, at least in principle. On the other hand, as Sho is not based on modern biology, the mechanism, or rather logic, of correspondence between Sho and Western disease entities may be very different from that between symptoms and diseases or formulae and diseases.

One important difference I wish to note explicitly is that in Kampo there is no distinction between mind and body. So almost all of the Sho concepts have elements which take into account the condition of the mind. Thus, the many-to-many correspondence between Sho and Western disease entities always comprises one important dimension, namely that of psychosomatic interrelationship. It may therefore not be too ambitious to predict that the Sho concept will help modern Western medicine to reorganize itself into more integrated mind-body medicine. Even in Japan, it is now not legitimate for a registered physician to prescribe any medicine because the patient exhibits, say, Kyo-Sho. It would be interesting to see if Western medical scientists explore the psychosomatic foundation of such Sho in the future, integrate it as a legitimate scientific term of future medicine, and then export it back to Japan.

Another important aspect of this many-to-many correspondence is that Kampo herbs are essentially multi-composite agents. Therefore, while, for example, aspirin's protean effects may be simply explained by its action on prostaglandin metabolism, it is possible that Kampo herbs are of Sho for multiple disease conditions exactly because each of them is a complex agent with various active edges. This is also a fundamental difference between modern Western medicine and Kampo.

\section{Reference}

1. Terasawa K. Evidence-based Reconstruction of Kampo Medicine: Part I-Is Kampo CAM? Evidence-based Complementary and Alternative Medicine 2004;1:11-16. 


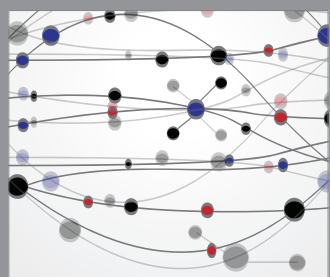

The Scientific World Journal
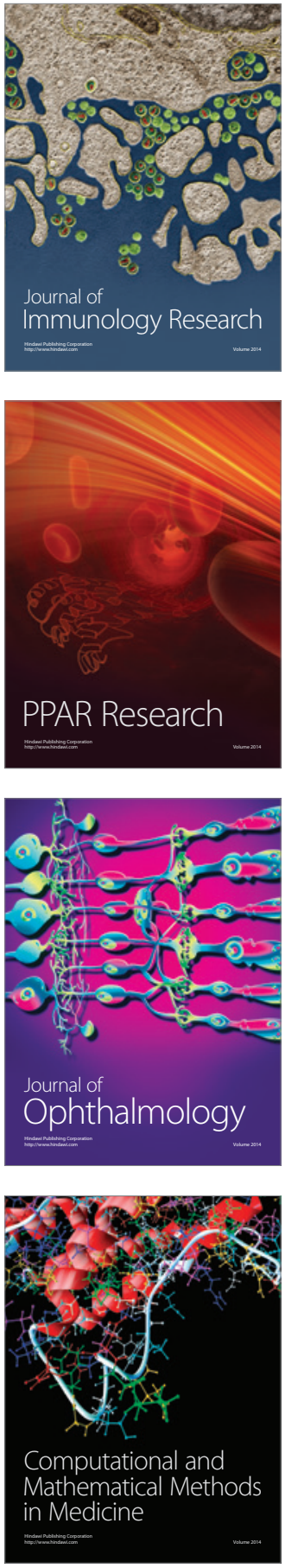

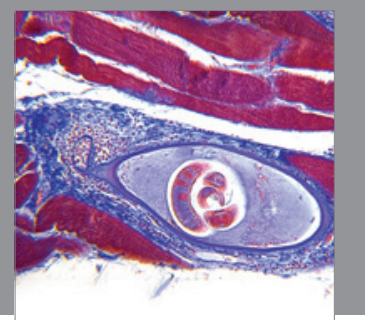

Gastroenterology

Research and Practice
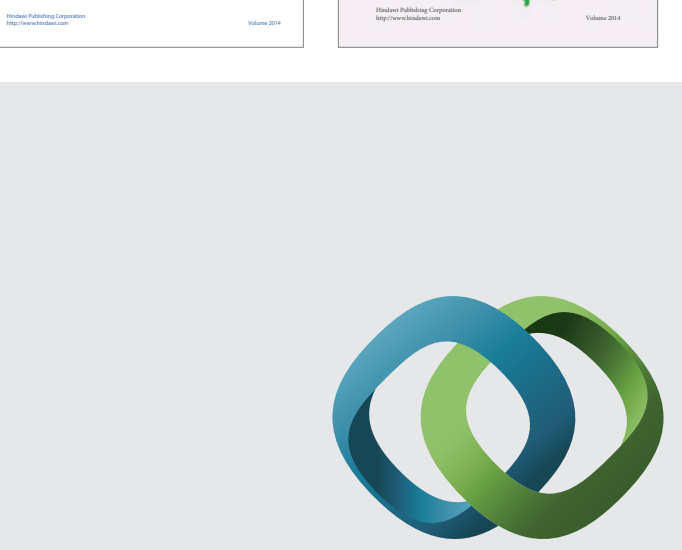

\section{Hindawi}

Submit your manuscripts at

http://www.hindawi.com
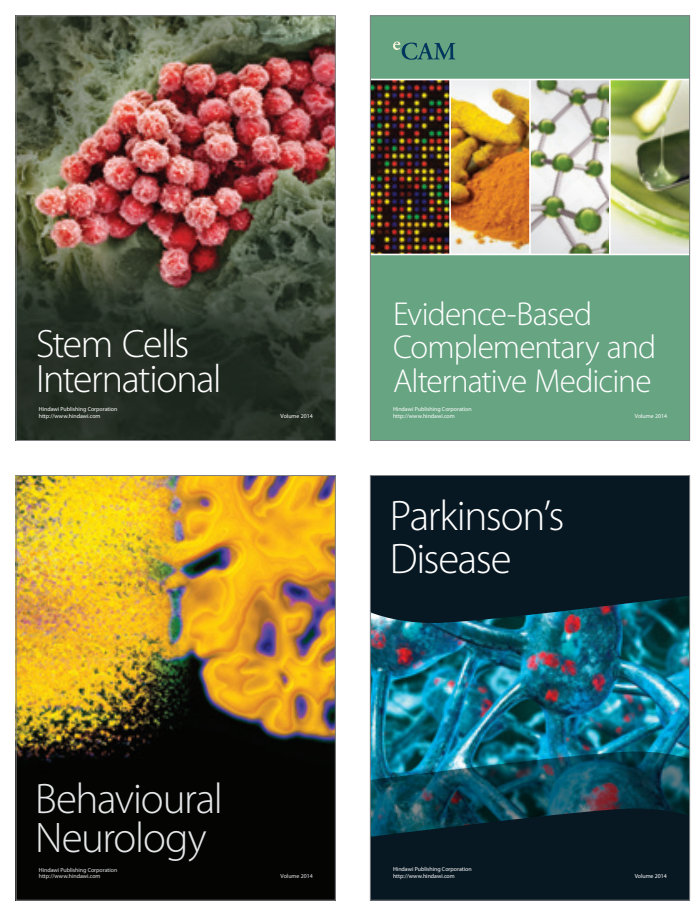

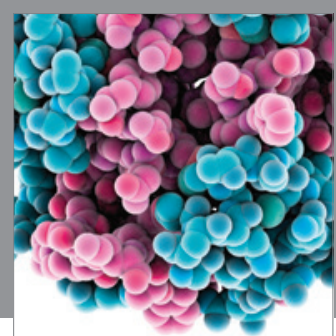

Journal of
Diabetes Research

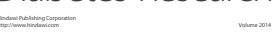

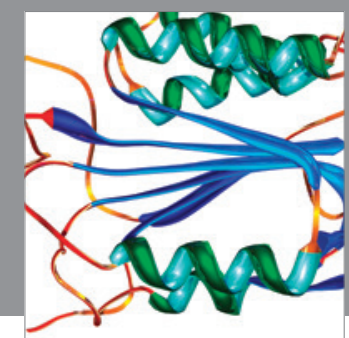

Disease Markers
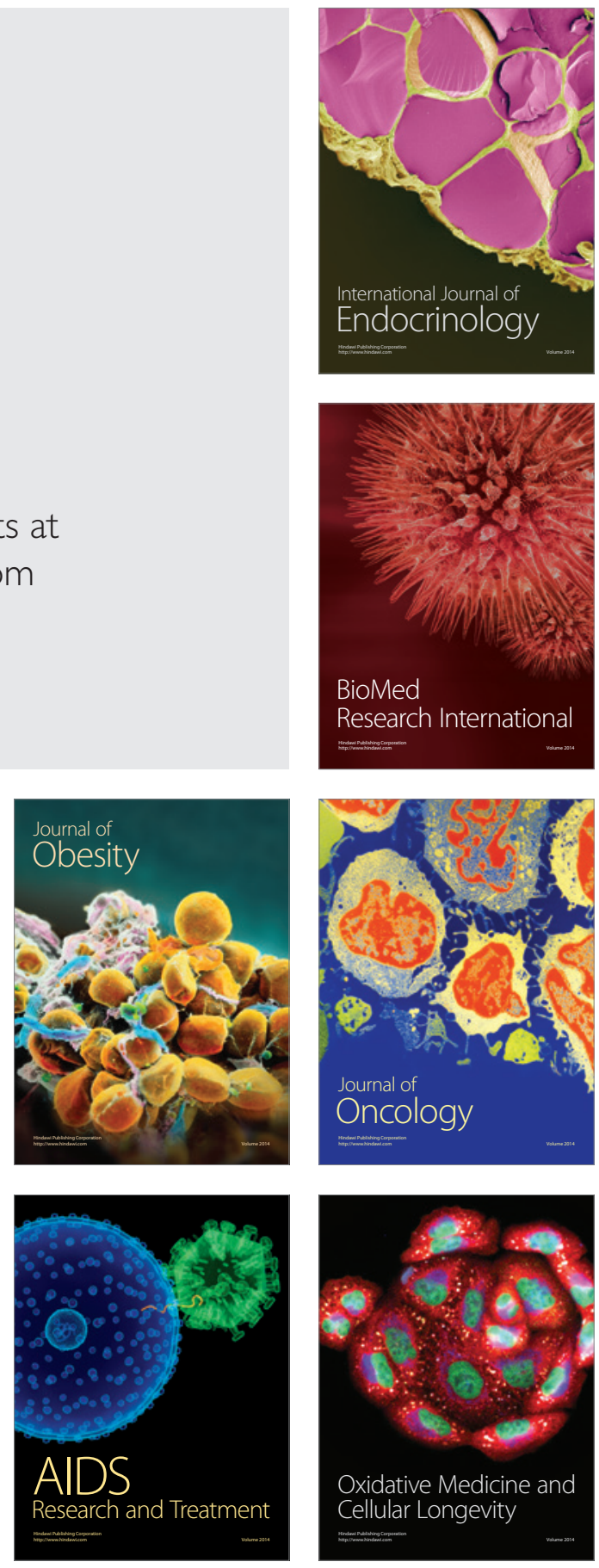